\title{
Serum Zonulin and Endotoxin Levels in Exceptional Longevity versus Precocious Myocardial Infarction
}

\author{
Pedro Carrera-Bastos ${ }^{1,2}$, Óscar Picazo², Maelán Fontes-Villalba ${ }^{1,2}$, Helios Pareja-Galeano ${ }^{3,4, *}$, \\ Staffan Lindeberg ${ }^{1}$, Manuel Martínez-Selles ${ }^{5}$, Alejandro Lucia ${ }^{3,4}$, Enzo Emanuele ${ }^{6}$ \\ ${ }^{1}$ Clinical Research Center, Faculty of Medicine, Center for Primary Health Care Research, Lund University, \\ Malmö, Sweden. \\ ${ }^{2}$ NutriScience - Education and Consulting, Lisbon, Portugal. \\ ${ }^{3}$ European University of Madrid, Spain. \\ ${ }^{4}$ Research Institute of Hospital 12 de Octubre ('i+12'), Madrid, Spain. \\ ${ }^{5}$ Servicio de Cardiología, Hospital Universitario Gregorio Marañón, CIBERCV, Universidad Europea, \\ Universidad Complutense, Madrid, Spain \\ ${ }^{6} 2 \mathrm{E}$ Science, Robbio, Pavia, Italy.
}

[Received March 23, 2017; Revised May 3, 2017; Accepted June 30, 2017]

\begin{abstract}
Endotoxemia-induced inflammation has been associated with insulin resistance and atherosclerosis, ultimately increasing the risk of coronary heart disease. Increased intestinal permeability is an important event leading to endotoxemia. This study aims to elucidate the possible association between endotoxin (lipopolysaccharide) and zonulin (a biomarker of intestinal permeability) levels and the risk of coronary heart disease, and thus healthy aging. Serum levels of zonulin, lipopolysaccharide and soluble CD14 (a protein that binds lipopolysaccharide) were measured in disease-free centenarians, young healthy controls and patients with precocious acute myocardial infarction. Disease-free centenarians had significantly lower levels of serum zonulin $(P<0.01)$ and lipopolysaccharide $(P<0.001)$ than young patients with acute myocardial infarction, and had significantly lower concentrations of serum lipopolysaccharide than young healthy controls $(P<0.05)$. No significant differences were found for soluble CD14 between groups. Our findings may stimulate further research into the role played by intestinal permeability and endotoxemia not only in coronary heart disease but also in lifespan modulation.
\end{abstract}

Key words: centenarians, longevity, inflammation, endotoxemia.

Low-grade chronic inflammation is associated with degenerative disorders including coronary heart disease (CHD), stroke and diabetes, which are among the leading causes of premature mortality in most regions of the world [1]. One possible cause of chronic inflammation is endotoxemia, a condition arising from absorption into circulation of the endotoxin lipopolysaccharide (LPS) [2], an active component of the cell wall of Gram-negative bacteria originating from the microbiota of the oral cavity and gut $[2,3]$ or from processed foods [4].

Translocation of LPS from the intestinal lumen into circulation can occur through transcellular or paracellular pathways [3], with the latter being regulated by a protein complex containing the enterocyte-derived protein

*Correspondence should be addressed to: Helios Pareja-Galeano, PhD, European University of Madrid, C/Tajo S/N, Urbanización El Bosque. Villaviciosa de Odón, Madrid, 28670 Spain. E-mail: helios.pareja@universidadeuropea.es

Copyright: () 2017 Carrera-Bastos, $\mathrm{P}$ et al. This is an open-access article distributed under the terms of the Creative Commons Attribution License, which permits unrestricted use, distribution, and reproduction in any medium, provided the original author and source are credited. 
zonulin [5]. Following specific stimuli (such as enteric bacteria and the wheat protein gliadin), zonulin reversibly disassembles intercellular tight junctions leading to increased intestinal permeability [5], which in turn may cause endotoxemia [6, 7] and low-grade chronic inflammation through activation of Toll-like receptors [7]. These phenomena are implicated in the pathogenesis of the metabolic syndrome, type 2 diabetes and CHD [2, $4,7,8]$. Accordingly, circulating zonulin and biomarkers of endotoxemia (LPS and LPS-binding protein) have been reported to be elevated in patients with CHD [6] and type 2 diabetes [7], and to be associated with insulin resistance $[7,9,10,11]$, dyslipidemia [7], inflammation [7, 9, 11], as well as with higher waist circumference, diastolic blood pressure, fasting glucose and increased risk of metabolic diseases [12]. Moreover, circulating LPS-binding protein is positively associated with atherosclerosis [13] and has been identified as an independent predictor of CHD [14] and cardiovascular mortality [15].

In light of the aforementioned evidence and of the fact that aging is potentially associated with an increased risk of insulin resistance [16], chronic inflammation [17] and CHD [18], as well as with increased levels of circulating zonulin [19] and LPS [20], we hypothesized that both a low intestinal permeability and endotoxemia may be associated with a lower risk of CHD and contribute to healthy longevity. We therefore measured serum zonulin, LPS and soluble CD14 (an acute phase protein that mediates immune responses to LPS [21]) in disease-free centenarians ("dodgers") [22, 23] and compared the results with young healthy controls and patients with precocious acute myocardial infarction (AMI).

\section{MATERIALS AND METHODS}

\section{Participants}

Three groups of subjects (described in previous publications [23-27]) were analyzed: disease-free centenarians ( $\mathrm{N}=79 ; 39$ men, 40 women), non-diabetic patients aged $<40$ years who had experienced AMI $(\mathrm{N}=178 ; 101$ men, 77 women), and healthy young volunteers matched to AMI patients for age and sex ( $\mathrm{N}=178 ; 102$ men, 76 women).

All subjects were Caucasian whites ascertained to be of Italian descent (Northern Italy, mainly from Lombardy and Piedmont). The centenarians were recruited mainly via general practitioners in the community and have been previously described [23, 26, 27]. The history of past and current diseases was accurately collected, checking the centenarians' medical documentation and the current drug therapy. Accordingly, all the centenarians were free of major age-related diseases (severe cognitive impairment, clinically evident cancer, CHD, renal insufficiency, or severe physical impairment). Healthy young controls were volunteers who were considered to be in good health, with exclusion criteria being as follows: presence of CHD or cerebrovascular disease, cancer, dementia, chronic autoimmune/inflammatory disorders, renal or hepatic failure, and major psychiatric conditions. Their compliance with study criteria was confirmed based on their family history and results of routine blood chemistry, i.e., none had hypercholesterolemia or hypertriglyceridemia. Further, none of them was obese or had hypertension, even though $36 \%$ were current smokers.

Table 1. Serum biochemical parameters in the three study groups.

\begin{tabular}{lcccc}
\hline \multicolumn{1}{c}{ Biomarkers } & Young AMI patients & Healthy young controls & Centenarians & $\boldsymbol{P}$-value \\
\hline LPS (EU/mL) & $15.4 \pm 5.2$ & $6.1 \pm 7.3$ & $4.2 \pm 5.3$ & $<0.001$ \\
Soluble CD14 (ng/mL) & $1,489 \pm 339$ & $1,438 \pm 312$ & $1,341 \pm 274$ & $\mathrm{~ns}$ \\
Zonulin (pg/mL) & $7.6 \pm 3.1$ & $5.2 \pm 2.7$ & $4.0 \pm 2.1$ & $<0.001$ \\
\hline
\end{tabular}

Abbreviations: AMI, acute myocardial infarction; LPS, lipopolysaccharide: ns, not significant $(P>0.05)$.

\section{Procedures and measures}

Fasting blood samples were drawn into EDTA tubes. LPS levels were measured with the QCL-1000® chromogenic Limulus amebocyte lysate endpoint assay (Lonza, Walkersville, MD, USA). Special care was taken to avoid LPS contamination of any solution or vessel. LPS content in serum was expressed as endotoxin units (EU) per $\mathrm{mL}$. Serum zonulin concentration was measured by quantitative ELISA (Immundiagnostik AG, Bensheim, Germany). Serum soluble CD14 was quantified using a commercially available ELISA (R\&D systems, Minneapolis, MN, USA). The minimum detectable limits for the biochemical tests were as follows: LPS, 0.1 $\mathrm{EU} / \mathrm{mL}$; zonulin, $0.1 \mathrm{pg} / \mathrm{mL}$; and soluble CD14: 125 $\mathrm{ng} / \mathrm{mL}$. Intra- and inter-assay coefficients of variation were as follows: LPS, $4.8 \%$ and $5.8 \%$; zonulin, $4.1 \%$ and $6.4 \%$; and soluble CD14, $4.1 \%$ and $6.0 \%$. 


\section{Statistical methods}

We compared zonulin and SCD14 levels between the three groups by one-way analysis of variance (ANOVA) followed by Tukey's post hoc test, and we used the Kruskal-Wallis test (which was skewed according to the
Shapiro-Wilk test) for LPS analysis. Correlations between the study variables were assessed with Spearman's rho test. All calculations were performed using SPSS (v17.0, SPSS Inc., Chicago, IL, USA) and GraphPad Prism (v6.0, GraphPad Inc., San Diego, CA, USA).

Table 2. Correlation coefficients (Spearman's $\rho$ ) between serum biomarkers in the three study groups.

\begin{tabular}{lllll}
\hline & & \multicolumn{1}{c}{ Endotoxin } & Soluble CD14 & Zonulin \\
\hline LPS & AMI & - & $\rho=-0.06, \mathrm{~ns}$ & $\rho=0.48, P<0.001$ \\
& Controls & - & $\rho=0.09, \mathrm{~ns}$ & $\rho=0.19, \mathrm{~ns}$ \\
& Centenarians & - & $\rho=-0.12, \mathrm{~ns}$ & $\rho=0.64, P<0.001$ \\
& AMI & $\rho=-0.06, \mathrm{~ns}$ & - & $\rho=0.12, \mathrm{~ns}$ \\
Soluble CD14 & Controls & $\rho=0.09, \mathrm{~ns}$ & - & $\rho=0.08, \mathrm{~ns}$ \\
& Centenarians & $\rho=-0.12, \mathrm{~ns}$ & - & $\rho=-0.01, \mathrm{~ns}$ \\
Zonulin & AMI & $\rho=0.48, P<0.001$ & $\mathrm{r}=0.12, \mathrm{~ns}$ & - \\
& Controls & $\rho=0.19, \mathrm{~ns}$ & $\mathrm{r}=0.08, \mathrm{~ns}$ & - \\
& Centenarians & $\rho=0.64, P<0.001$ & $\mathrm{r}=-0.01, \mathrm{~ns}$ & - \\
\hline
\end{tabular}

Abbreviations: AMI, acute myocardial infarction; LPS, lipopolysaccharide; ns, not significant $(P>0.05)$.

\section{RESULTS}

As shown in Table 1, LPS levels were significantly lower in disease-free centenarians than in AMI patients ( $P<0.001$, post-hoc Dunn's test after Kruskal-Wallis test) and healthy young controls $(P<0.05$, post-hoc Dunn's test after Kruskal-Wallis test). Zonulin levels were significantly lower in centenarians than in AMI patients $(P<0.01)$, but no differences were found when centenarians were compared with healthy controls $(P>0.05$, post-hoc Tukey's test after ANOVA). No significant differences were found between the groups in the levels of soluble CD14 (Table 1) $(P>0.05)$. The aforementioned results remained essentially unchanged when analyzing men and women separately (data not shown). On the other hand, LPS levels correlated with those of zonulin within the group of centenarians $(\rho=0.64$, $P<0.001)$ and AMI patients $(\rho=0.48, P<0.001)$, but not within healthy young controls $(\rho=0.19, P>0.05)$ (Table 2$)$.

\section{DISCUSSION}

The present study shows that disease-free centenarians have lower serum zonulin and LPS levels than young AMI patients. Moreover, the two variables correlate with each other in centenarians and AMI patients, suggesting that intestinal hyperpermeability may cause endotoxemia, which in turn could lead to inflammation and hence insulin resistance, atherosclerosis and hypercoagulation $[2,4,7,8,28,29]$. Regarding soluble CD14, although its levels have been found to be higher in patients with unstable angina [30], in individuals with the metabolic syndrome (which in turn is associated with an increased CHD risk) [21], and in HIV-infected patients (in whom CD14 levels are correlated with atherosclerosis [31] and coronary artery calcification [32]), no significant intergroup differences were found in the present study. Echoing our findings, other studies have yielded similar negative results $[33,34]$. Thus, although more research is needed, our data suggest that serum levels of zonulin and LPS emerge as potential novel biomarkers of exceptional longevity. Indeed, the present cohort of centenarians has been the subject of previous research identifying other potential serum biomarkers of healthy exceptional longevity and thus of successful aging, i.e., irisin [23], vitamin D [24], eicosapentaenoic acid to arachidonic acid ratio [25], beclin-1 [26], or the combination of chemerin, fetuin-A, and fibroblast growth factors 19 and 21 [27].

Our data, coupled with the fact that aging per se is typically associated with elevated circulating zonulin [19], endotoxemia [20], inflammation [17], insulin resistance [16] and atherosclerosis [18], suggest that 

LPS translocation may help to reduce the risk of CHD and contribute to healthy lifespan. Of interest, zonulin is identical to the precursor of haptoglobin [5], an acutephase protein that scavenges the potent oxidant free hemoglobin [5] and has anti-inflammatory activity [35]. Moreover, genetic variants of the haptoglobin gene have been associated with CHD [36] and longevity [35].

Experimental manipulation of zonulin and endotoxemia in animal models of aging and AMI, and human intervention studies are required before definite conclusions can be drawn as reverse causality cannot be firmly excluded in our study giving its observational nature. Another limitation is the lack of data on subjects' diet and microbiota composition, since these are suspected environmental triggers of endotoxemia and zonulin expression. While we cannot yet translate our findings into clinical practice, we hope that they may inspire future studies to establish a causal link between intestinal permeability, endotoxemia and CHD.

\section{Acknowledgments}

We wish to thank all participants for their cooperation. Research by Alejandro Lucia is funded by Fondo de Investigaciones Sanitarias (FIS, grant \# PI15/00558) and Fondos FEDER" and "We thank Dr. Staffan Lindeberg, who has unfortunately passed away, for his friendship and collaboration and for the effort he put into this paper despite being severely ill. Our deepest condolences to his family and close friends.

\section{Conflict of Interest}

The authors have no financial, personal or other potential conflicts of interest to declare.

\section{References}

[1] Mortality GBD, Causes of Death C (2016). Global, regional, and national life expectancy, all-cause mortality, and cause-specific mortality for 249 causes of death, 1980-2015: a systematic analysis for the Global Burden of Disease Study 2015. Lancet, 388: 1459-1544

[2] Patel PN, Shah RY, Ferguson JF, Reilly MP (2015). Human experimental endotoxemia in modeling the pathophysiology, genomics, and therapeutics of innate immunity in complex cardiometabolic diseases. Arterioscler Thromb Vasc Biol, 35: 525-534

[3] Kelly CJ, Colgan SP, Frank DN (2012). Of microbes and meals: the health consequences of dietary endotoxemia. Nutrition in clinical practice : official publication of the American Society for Parenteral and Enteral Nutrition, 27: 215-225 measures taken to decrease intestinal permeability and Dietz S, Winkelmann BR, et al. (2011). Lipopolysaccharide-binding protein (LBP) is associated with total and cardiovascular mortality in individuals with or without stable coronary artery disease--results from the Ludwigshafen Risk and Cardiovascular Health Study (LURIC). Atherosclerosis, 219: 291-297 dietary intake of pro-inflammatory Toll-like receptor stimulants favourably modifies markers of cardiometabolic risk in healthy men. Nutr Metab Cardiovasc Dis, 26: 194-200

Fasano A (2011). Zonulin and its regulation of intestinal barrier function: the biological door to inflammation, autoimmunity, and cancer. Physiol Rev, 91: 151-175 (2016). Zonulin Regulates Intestinal Permeability and Facilitates Enteric Bacteria Permeation in Coronary Artery Disease. Sci Rep, 6: 29142

, , Lakshmi BS, et al. (2014). proinflammation in patients with type 2 diabetes. Mol Cell Biochem, 388: 203-210

Carrera-Bastos P, Fontes-Villalba M, O'Keefe JH, Lindeberg S, Cordain L (2011). The western diet and lifestyle and diseases of civilization. Res Rep Clin

, Waget A, Pardo G, et al. (2012). Circulating lipopolysaccharide-binding protein (LBP) as a marker 36: 1442-1449

Moreno-Navarrete JM, Sabater M, Ortega F, Ricart W, Fernandez-Real JM (2012). Circulating zonulin, a marker of intestinal permeability, is increased in association with obesity-associated insulin resistance. PLoS One, 7: e37160

Mokkala K, Pellonperä O, Röytiö H, Pussinen P, Ronnemaa T, Laitinen K (2017). Increased intestinal pernatity, measured by serum zonulin, is pregnant women. Metabolism 69, 43-50

Ohlsson B, Orho-Melander M, Nilsson PM (2017). Higher levels of serum zonulin may rather be associated with increased risk of obesity and hyperlipidemia, than with gastrointestinal symptoms or disease manifestations. Int J Mol Sci, 18(3): pii: E582

Serrano M, Moreno-Navarrete JM, Puig J, Moreno M, Guerra E, Ortega F, et al. (2013). Serum atherosclerosis. Atherosclerosis, 230: 223-227

Lepper PM, Schumann C, Triantafilou K, Rasche FM, Schuster T, Frank H, et al. (2007). Association of lipopolysaccharide-binding protein and coronary artery disease in men. J Am Coll Cardiol, 50: 25-31

\section{Lepper PM, Kleber ME, Grammer TB, Hoffmann K,}

Herieka M, Faraj TA, Erridge C (2016). Reduced (LPS) circulatory levels of lipopolysaccharide 
[16] Moller N, Gormsen L, Fuglsang J, Gjedsted J (2003). Effects of ageing on insulin secretion and action. Horm Res, 60: 102-104

[17] Bruunsgaard H, Pedersen BK (2003). Age-related inflammatory cytokines and disease. Immunol Allergy Clin North Am, 23: 15-39

[18] Niccoli T, Partridge L (2012). Ageing as a risk factor for disease. Current biology : CB, 22: R741-752

[19] Zak-Golab A, Kocelak P, Aptekorz M, Zientara M, Juszczyk L, Martirosian G, et al. (2013). Gut microbiota, microinflammation, metabolic profile, and zonulin concentration in obese and normal weight subjects. Jnt J Endocrinol, 2013: 674106

[20] Ghosh S, Lertwattanarak R, Garduno Jde J, Galeana JJ, Li J, Zamarripa F, et al. (2015). Elevated muscle TLR4 expression and metabolic endotoxemia in human aging. J Gerontol A Biol Sci Med Sci, 70: 232246

[21] Jialal I, Rajamani U, Adams-Huet B, Kaur H (2014). Circulating pathogen-associated molecular pattern binding proteins and High Mobility Group Box protein 1 in nascent metabolic syndrome: implications for cellular Toll-like receptor activity. Atherosclerosis, 236: $182-187$

[22] Geroldi D, Falcone C, Minoretti P, Emanuele E, Arra M, D'Angelo A (2006). High levels of soluble receptor for advanced glycation end products may be a marker of extreme longevity in humans. J Am Geriatr Soc, 54: 1149-1150

[23] Emanuele E, Minoretti P, Pareja-Galeano H, SanchisGomar F, Garatachea N, Lucia A (2014). Serum irisin levels, precocious myocardial infarction, and healthy exceptional longevity. Am J Med, 127: 888-890

[24] Pareja-Galeano H, Alis R, Sanchis-Gomar F, Lucia A, Emanuele E (2015). Vitamin D, precocious acute myocardial infarction, and exceptional longevity. Int $\mathrm{J}$ Cardiol, 199: 405-406

[25] Pareja-Galeano H, Sanchis-Gomar F, Santos-Lozano A, Garatachea N, Fiuza-Luces C, Lucia A, et al. (2015). Serum eicosapentaenoic acid to arachidonic acid ratio is associated with cardio-healthy exceptional longevity. Int J Cardiol, 184: 655-656

[26] Emanuele E, Minoretti P, Sanchis-Gomar F, ParejaGaleano H, Yilmaz Y, Garatachea N, et al. (2014). Can enhanced autophagy be associated with human longevity? Serum levels of the autophagy biomarker beclin-1 are increased in healthy centenarians. Rejuvenation Res, 17: 518-524

[27] Sanchis-Gomar F, Pareja-Galeano H, Santos-Lozano A, Garatachea N, Fiuza-Luces C, Venturini L, et al.
(2015). A preliminary candidate approach identifies the combination of chemerin, fetuin-A, and fibroblast growth factors 19 and 21 as a potential biomarker panel of successful aging. Age (Dordr), 37: 9776

[28] Ferguson JF, Shah RY, Shah R, Mehta NN, Rickels MR, Reilly MP (2015). Activation of innate immunity modulates insulin sensitivity, glucose effectiveness and pancreatic beta-cell function in both African ancestry and European ancestry healthy humans. Metabolism, 64: 513-520

[29] Bester J, Pretorius E (2016). Effects of IL-1beta, IL-6 and IL-8 on erythrocytes, platelets and clot viscoelasticity. Sci Rep, 6: 32188

[30] Zalai CV, Kolodziejczyk MD, Pilarski L, Christov A, Nation PN, Lundstrom-Hobman M, et al. (2001). Increased circulating monocyte activation in patients with unstable coronary syndromes. J Am Coll Cardiol, 38: $1340-1347$

[31] McKibben RA, Margolick JB, Grinspoon S, Li X, Palella FJ, Jr., Kingsley LA, et al. (2015). Elevated levels of monocyte activation markers are associated with subclinical atherosclerosis in men with and those without HIV infection. J Infect Dis, 211: 1219-1228

[32] Longenecker CT, Jiang Y, Orringer CE, Gilkeson RC, Debanne S, Funderburg NT, et al. (2014). Soluble CD14 is independently associated with coronary calcification and extent of subclinical vascular disease in treated HIV infection. Aids, 28: 969-977

[33] Koenig W, Khuseyinova N, Hoffmann MM, Marz W, Frohlich M, Hoffmeister A, et al. (2002). CD14 C(260)-->T polymorphism, plasma levels of the soluble endotoxin receptor CD14, their association with chronic infections and risk of stable coronary artery disease. J Am Coll Cardiol, 40: 34-42

[34] Gonzalez-Quintela A, Alonso M, Campos J, Vizcaino L, Loidi L, Gude F (2013). Determinants of serum concentrations of lipopolysaccharide-binding protein (LBP) in the adult population: the role of obesity. PLoS One, 8: e54600

[35] Napolioni V, Gianni P, Carpi FM, Concetti F, Lucarini N (2011). Haptoglobin (HP) polymorphisms and human longevity: a cross-sectional association study in a Central Italy population. Clin Chim Acta, 412: 574-577

[36] Horne BD, Anderson JL (2015). Haptoglobin 2-2 Genotyping for Refining Standard Cardiovascular Risk Assessment: A Promising Proposition in Need of Validation. J Am Coll Cardiol, 66: 1800-1802 